\title{
Use of Software Defined Radio Receivers for Antenna Measurements
}

\author{
Rubén T. Sánchez, Manuel Sierra Castañer, and L. J. Foged
}

\begin{abstract}
This paper presents a time domain antenna measurement technique that aims to measure antennas in referenceless scenarios. The technique resolves measurement challenges derived from antennas where the reference signal is not accessible, for intance in EMC or active antennas. The algorithm implemented in this work is based on the the Fast Fourier Transform of the time domain signal to estimate the power spectrum and the relative phase between measurement points. In order to do that a reference antenna is used to retrieve the phase, providing a full characterization in amplitude and phase of the electric field and allowing source reconstruction. The results demonstrate the potential of this technique for new antenna measurement systems and reveal some of the limitations of the technique to be optimized, like the undesired reflections due to the interactions between the probe and the reference antenna.
\end{abstract}

\section{INTRODUCTION}

The development of new technologies like the ones derived from the evolution of the fifth generation (5G) implies more complex devices. The complexity of the devices can be seen in the necessity of technologies as phased arrays, massive multiple-input multiple-output (MIMO) or smart antennas, along with others. Therefore, the antenna will not be conceived as a device but as a system.

Antenna measurement techniques will have to change in order to adapt to this revolution. In the future, the systems will be so compact that there will be no room for conventional connectors. In [1] a description of the challenges for on-chip-antennas (OCA) is studied. The overview describes how even when there is room for a radio frequency (RF) connector between the antenna and the radio system it will be cost-ineffective. Massive MIMO is a clear example where hundreds of antennas may be used and a RF connector for each antenna would be very costly. In [2] the challenges when coaxial feeding is not used are described, since for integrated antennas it will be only possible to probe input and output signals.

Along with the architecture challenges, the higher frequency will require very precise acquisition systems. Considering the future challenges derived from the characteristics of the antenna systems, over the air measurements (OTA) will play an important role to characterize the performance of the antenna, since traditional tests performed using coaxial cables to obtain the reference will not be possible.

Another clear application of referenceless measurements can be found in EMC, the importance of characterizing and evaluating the impact of the non-intentional radation lies not only in power measurements but also in phase, since source reconstruction and evaluation of the field at different distances is possible.

Therefore, besides the far field pattern of the antenna, a very important part of the measurement is the diagnostic of the AUT: processing the data collected from the AUT in the near-field and identification of possible irregularities in the radiation of the device. These techniques require the acquisition of the electric field in amplitude and phase.

Thus it is clear that there are applications where the phase reference can not be obtained in order to acquire the near-field information. There is a wide range of techniques that have been applied in the literature regarding phaseless measurements; these methods are suitable for scenarios where the phase is not accessible. Holographic methods can be seen in [3-8]; this method requires only one scan surface, but it requires further hardware for a reference field. In addition, due to overlapping in the spatial domain it may require a high sampling rate. Iterative methods between two surfaces have been tested for planar [9], cylindrical [10] and spherical [11] configurations as well; this method introduces complexity to the measurement process since two scanning surfaces are needed. Furthermore, the method has not generally an unique solution and it requires that the propagation of the field provides enough information in the two surfaces. Interferometry is another existing method [12]; the technique requires the development of further hardware to obtain the power of the in-phase and in-quadrature combinations of two probe signals. 
Thus, considering the existing limitations in cost (due to extra hardware), and time (if more than one surface is used), it is essential to implement an accurate and cost-effective technique for antenna characterization in referenceless scenarios. This work proposed a simple and costeffective alternative method in comparison with the existing techniques. The method is based on the improvements of software defined radio (SDR) receivers architectures that allows OTA time domain measurements of the desired signals.

The paper is organized as follows: in Section 2 an explanation of the SDR system, the algorithm and the limitations will be given. Section 3 will give some measurement results of the normalized radiation patterns at 2 and $5.75 \mathrm{GHz}$ when using the proposed measurement technique. Section 4 will describe a preliminary spherical near-field test as a step towards the final optimization of the system. Finally, in Section 5 the conclusions and future lines are presented.

\section{RECEIVER SPECIFICATION AND RETRIEVAL ALGORITHM}

\subsection{SDR Specification}

In order to implement the low-cost time domain phaseless technique the Zynq Evaluation and Development Board (ZedBoard) in conjunction with the transceiver AD-FMCOMMS3-EBZ was used. The radio frequency integrated transceiver provides a configurable digital interface to a FPGA, and that is how the communication is established between the ZedBoard and the RF module.

The $2 \times 2$ transceiver module consists of a 12 bit $\mathrm{ADC}$ with a receiver band that goes from $70 \mathrm{MHz}$ to $6 \mathrm{GHz}$ and a tunable channel bandwidth up to $56 \mathrm{MHz}$. The receiver is homodyne, meaning that the signal is directly down converted to base-band for digitization purposes, more specifically IQsamples are generated by the transceiver module. The receiver chain can be controlled and it consists of two programmable low-pass filters, decimating filters and gain control for each channel.

One of the limitations of the board is the acquisition system. The system performs single batch captures in a ring buffer structure. That means that to keep up with the probe position the buffer must be flushed.

\subsection{Power and Phase Algorithm}

The calculation of the amplitude and phase is based on the FFT analysis of the signal received. Fig. 1 shows the process followed to estimate the power and phase of the measured electric field. The filtering included in the SDR is programmed to be a matched filter with respect to the sampling frequency, so that the noise is not replicated inside the band under interest.

Following with the conditioning of the signal a Blackman-Harris window is applied to the input data before computing the FFT. The performance of this window is suitable for the measurement of tones, and this is exactly what is implemented in the tests carried out.

Once the signal is well conditioned the power and phase calculation are done. The power calculation is based on Parseval's theorem, therefore the power is calculated as expressed in Eq. (1). The right-hand side of Eq. (1) is integrated around the maximum since single frequency transmission

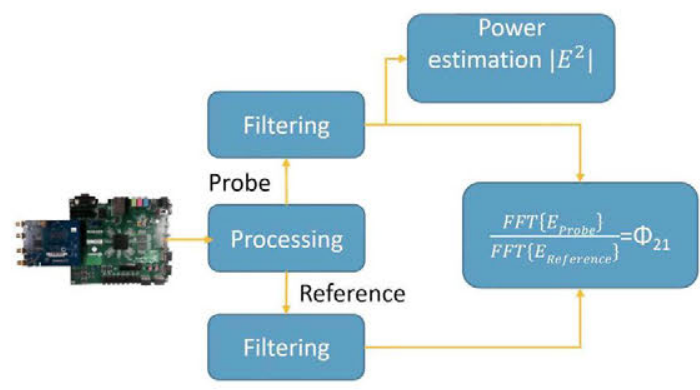

Figure 1: Phase and power estimation.

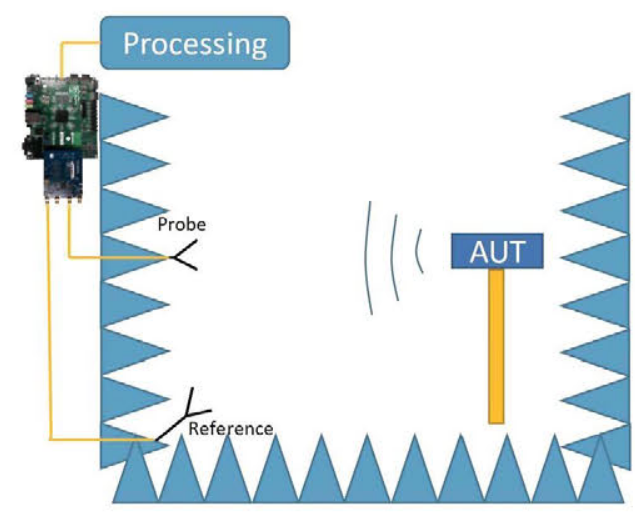

Figure 2: Antenna measurement set-up for phase reconstruction. 
is being analyzed.

$$
\sum_{n=0}^{N-1}|x(n)|^{2}=\frac{1}{N} \sum_{k=0}^{N-1}|X(k)|^{2}
$$

The phase retrieval is based on the the ratio between the Fast Fourier Transform (FFT) of the reference and the probe channels (see Eq. (2)). Since there could be slight differences in the peak of the two channels the phase is calculated as the ratio of the FFT's for the maximums of each channel.

$$
\phi_{12}=\operatorname{angle}\left(\frac{\left.\mathcal{F}\left(E_{\text {probe }}\right)\right)}{\mathcal{F}\left(E_{\text {reference }}\right)}\right)
$$

This method for retrieving the phase takes into account possible drifts of the transmitted signal, since the reference is obtained by measuring the same signal received by the probe.

\subsection{Limitations}

If the effect of the reference is not considered and only errors due to the SDR are analyzed, the limitations are mainly attributed to the SNR and IQ-imbalance.

The IQ-imbalance is a well-known limitation of the SDR. In the particular case of the ADFMCOMMS3-EBZ it is automatically compensated by an iterative intern algorithm. This removes the necessity of implementing an algorithm to compensate for imbalanced IQ-channels.

Regarding the noise, some simulations and measurements were carried out in the laboratory. First, the noise in each of the IQ-channels was modeled as additive white Gaussian noise (AWGN). The power of the noise was chosen depending on the SNR to be evaluated. In that way, the uncertainty due to the noise effect is isolated. Afterwards, some measurements of the SDR were done in order to derive the statistics of the error intruduced in amplitude and phase when a tone is measured in presence of noise and IQ-imbalance. The measurement uncertainties were analyzed for a frequency range that goes from 1 to $6 \mathrm{GHz}$ in steps of $0.25 \mathrm{GHz}$. The results were obtained by comparing the results of the received signal by the SDR and the vector network analyzer (VNA). The exhaustive study is not included here, but the conclusions derived are given in this paper:

- The dynamic range of the SDR is about $50 \mathrm{~dB}$.

- In the wost case scenario, peak errors of $\pm 12^{\circ}$ in phase and $\pm 1.3 \mathrm{~dB}$ in amplitude may be found.

- The dynamic range may be improved by measuring more samples, since the noise is bandlimited.

The measurement time is another important factor to optimize if multifrequency scenarios are considered. The acquisition time of the SDR after optimizing the amount of information measured is about $0.2 \mathrm{~s}$. The optimization of this factor represents a trade-off between uncertainty and saving time, different situations are considered in Section 3.

\section{MEASUREMENT TEST}

\subsection{Measurement Set-up}

The set-up is composed by two probe antennas and the AUT. One of the antennas is the conventional probe used for near-field antenna measurements, the other one is a reference that will be used to get the phase reference for the measurements. Fig. 2 shows the set-up of the measurement system for a planar near field scanner; the AUT is in transmitting mode, then the probe and the reference are feeding the two receiving channels of the SDR.

The first thing to notice is the position of the reference antenna, it have to be placed in a fixed position with respect to the AUT. Moreover, the reference antenna should be placed in a position where the signal received is strong enough to ensure reliability in the measurements, and where the interference with the system is not amplified. It will be shown how post-processing techniques may be applied to remove the interference of the reference antenna. In this section, two different measurements will be evaluated at different frequencies, 2 and $5.75 \mathrm{GHz}$. The measurements will be perform in the LEHA-UPM facility. These measurements will provide information about the limitations of this method and some conclusions about the potential of the technique. 


\subsection{Planar Near Field Measurement at 2 and $5.75 \mathrm{GHz}$}

The geometry of the measurement for the set-up at $2 \mathrm{GHz}$ can be seen in Fig. 3. The figure shows the planar coordinate system where the probe is moved and the $x$ and $y$-axis of the AUT coordinate system. The selected AUT was a standard gain horn (SGH) antenna. The probe is a single-port conical horn antenna with a broad radiation pattern. The reference is a pyramidal horn antenna. Before performing the measurement process some considerations were taken:

- The reference antenna was placed in a position where the received power was high enough to minimize uncertainty due to instability in the signal measured.

- The AUT, the probe, and the reference are linear polarized antennas. Only the $x$-component of the field was measured since the $y$-component is negligible over the scanning surface.

- A calibration was performed before the measurement in order to adjust the plane size according to the dynamic range of the SDR $(50 \mathrm{~dB})$. Finally, the planar scanner size was $2.3 \mathrm{~m}$. The distance between the AUT and the probe was fixed to $0.8 \mathrm{~m}$ (trade-off between multiple reflections and reliable region). With this information and the maximum size of the AUT $(0.37 \mathrm{~m})$ the reliable region is about $\pm 50^{\circ}$.

- The step chosen in $x$ and $y$-axis was $\frac{\lambda}{2}$.

- For each measurement point the amount of information captured was 40000 samples. This represented a good trade-off in terms of noise rejection.

After processing the measurements some deep ripples were observed in the radiation pattern. It was thought that most likely the ripples observed in the measured data were a consequence of the coupling between the probe and the reference antenna, therefore post-processing techniques were applied to the measurements.

From all the existing techniques in the literature, spatial filtering was the one chosen (see [13]). The method is based on considering the reflections as if they were generated by virtual sources over the AUT plane, so the idea is to filter out the undesired components once the field has been backpropagated to the AUT plane. The post-processing starts by calculating the plane wave spectrum (PWS) of the measured electric field, then it is back-propagated to the aperture plane and finally the undesired sources of the electric field are filtered out. Fig. 4 shows the reconstructed electric field after back-propagating to the AUT plane. The reflections can be clearly seen around the physical size of the AUT (red square in the figure). The spatial filtering will not only remove this effect but also reduce the noise of the measurement as can be seen in [14].

The effect of the probe should also be considered in order to compare the radiation pattern of the AUT with the reference. The probe is considered to be ideal in terms of cross-polarization rejection, then if the far-field radiation pattern of the probe is known the probe correction can be applied.

The $x$ and $y$-axis of the coordinate system of the radiation pattern are shown in Fig. 3. The $z$-axis is in the direction of the main beam. The co-polar and cross-polar unit vectors correspond

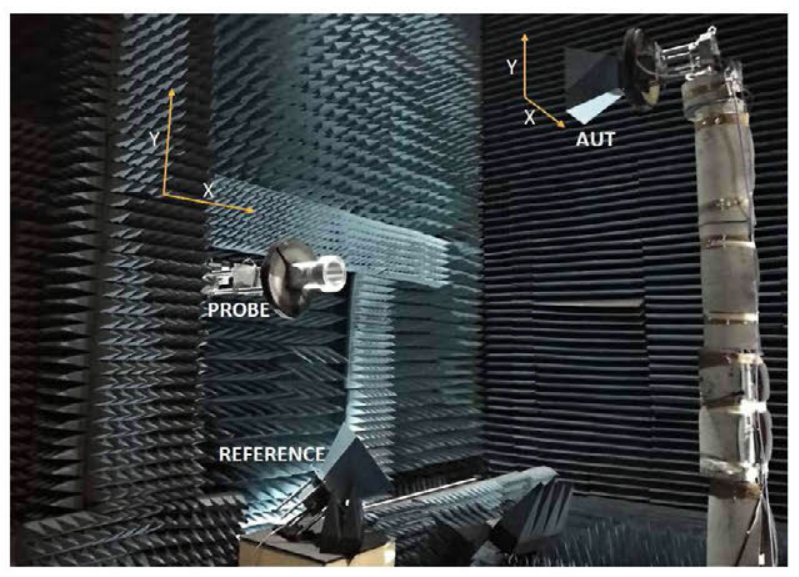

Figure 3: Measurement set-up at $2 \mathrm{GHz}$.

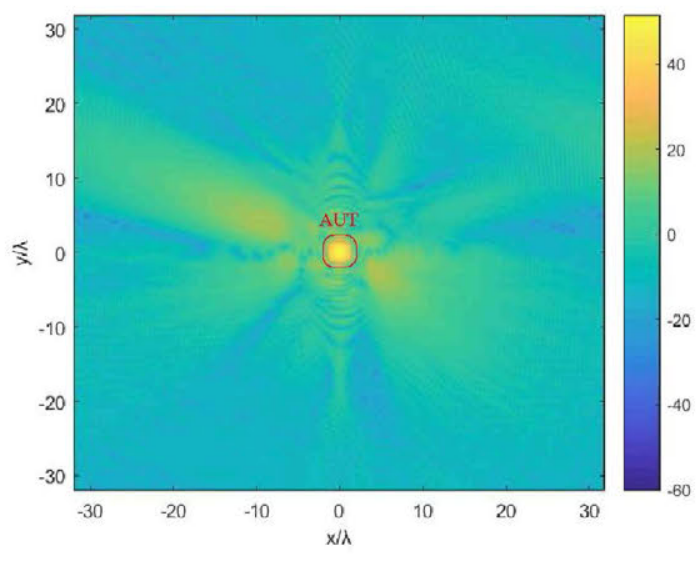

Figure 4: Reconstructed electric field at the aperture plane of the AUT. 
to Ludwig's 3rd definition [15], in such a way that the maximum of the electric field is oriented in the $x$-direction on the $z$-axis.

The radiation patterns for the main planes, $\phi=0^{\circ}$ and $\phi=90^{\circ}$ can be seen in Figs. 5 and 6. In the figures the reference is compared with the radiation pattern measured and the radiation pattern after post-processing (spatial filtering and probe compensation). It can be observed that the position of the reference during the measurement process is critical since the post-processing technique has removed the deep ripples observed in the measurements.

Despite some differences observed for the side lobes of $\phi=0^{\circ}$ cut, there is a good agreement between the radiation pattern measured after processing and the reference. The differences observed may be due to the size and the position of the reference antenna since it was not optimized in terms of reflections.

In order to better evaluate the performance of the SDR receiver, another test was performed at $5.75 \mathrm{GHz}$ to test the SDR at the limit of the maximum operation frequency and trying to reduce the effect of the reflections (see Fig. 7). In this case the reference was placed in a further position with respect to the AUT and the probe antenna, trying to minimize the reflections observed in Section 3.2. The AUT is again a SGH antenna and the probe antenna has a broad radiation pattern. Nevertheless, in this test it was decided not to consider probe compensation and the far-field obtained with this technique and the far-field obtained by measuring with a conventional planar near-field configuration (without the reference interfering) are compared instead. Thus, two planar near-field measurements were carried out, one conventional measurement and another one by using the new technique.

The following considerations were taken for this measurement in comparison to the one at $2 \mathrm{GHz}$ :

- The size of the measurement plane was set to $2 \times 2 \mathrm{~m}$.

- The distance between the AUT and the probe was fixed to $0.5 \mathrm{~m}$.

- The reliable region is $\pm 60^{\circ}$.

- The measurement time was pushed to $0.2 \mathrm{~s}$ for each measurement point (4000 samples).

The comparison between the near-field measurement results by using the SDR as a receiver to recover the electric field and the VNA showed that the differences in amplitude were below $-40 \mathrm{~dB}$ for all the measurement points. It was observed that the decrease in the amount of information measured (10 times less information in comparison with the previous test) worsen the dynamic range by about $10 \mathrm{~dB}$. There were still some differences observed for the phase, these differences may be attributed not only to the uncertainty of the receiver but also to the reflections from the AUT. It was difficult to remove this effect since the position was not completely optimized. Therefore not only the energy reflected to the AUT from the reference is an issue but also the scattered energy that interferes with the reference and changes the phase stability along the measurement.

Post-processing was also applied in this test not only to minimize the possible reflections but also to minimize noise in the measurement. Figs. 8 and 9 show the good agreement between

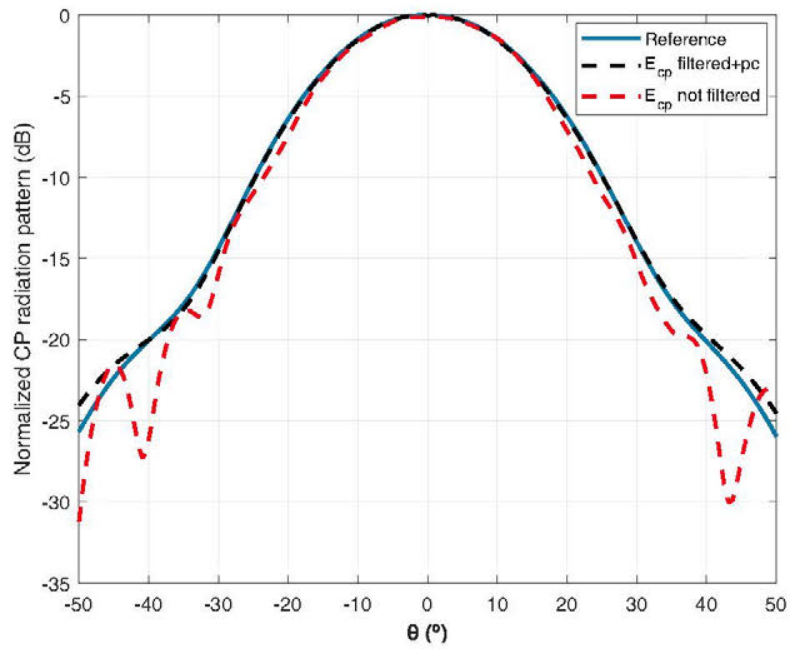

Figure 5: Normalized radiation pattern $\phi=90^{\circ}$ at $2 \mathrm{GHz}$.

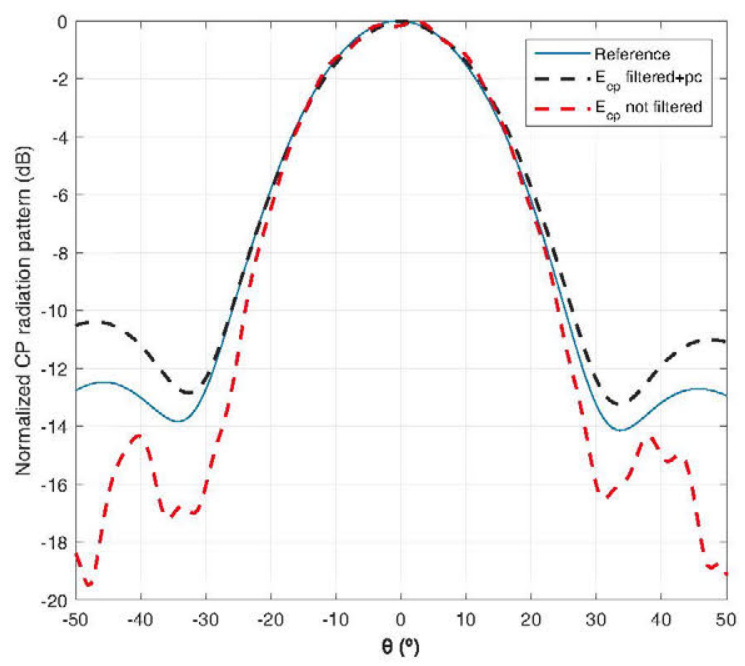

Figure 6: Normalized radiation pattern $\phi=0^{\circ}$ at $2 \mathrm{GHz}$. 


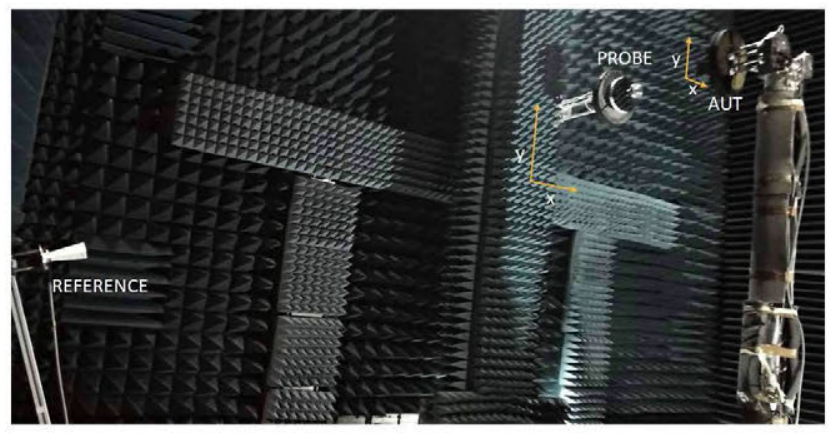

Figure 7: Measurement set-up at $5.75 \mathrm{GHz}$.

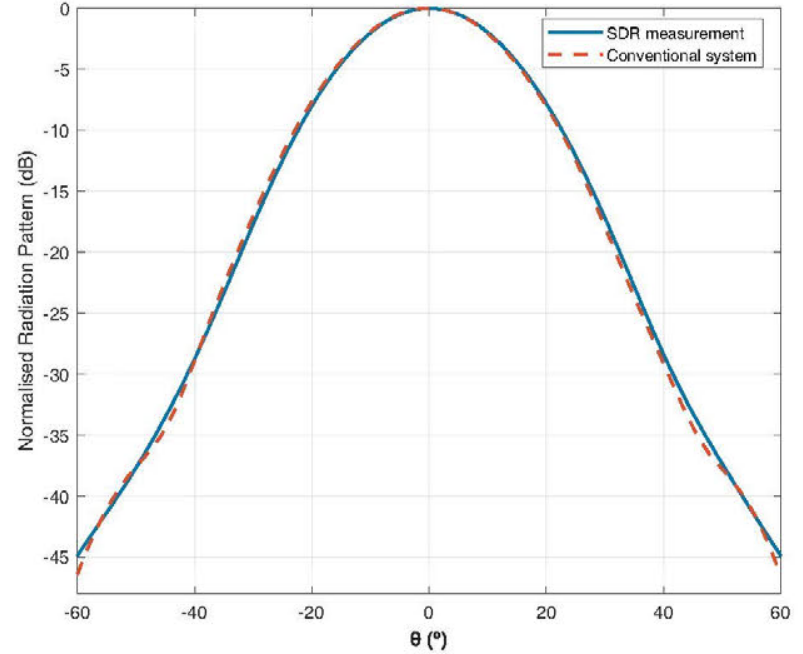

Figure 8: Normalized radiation pattern $\phi=90^{\circ}$ at $5.75 \mathrm{GHz}$.

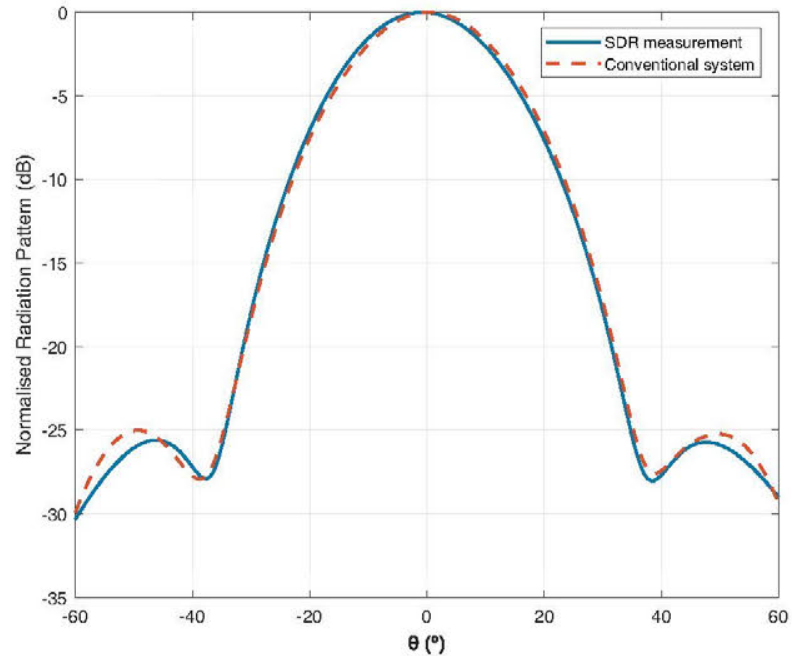

Figure 9: Normalized radiation pattern $\phi=0^{\circ}$ at $5.75 \mathrm{GHz}$.

the conventional planar near-field measurement when using the VNA and the measurement results when using the proposed technique in this paper after far-field transforming.

There are some conclusions about the proposed measurement method that can be derived from the previous results:

- The method is sensitive to the location and size of the reference antenna since coupling may create distortion in the amplitude measured or in the phase reference.

- The amount of information measured is a critical factor in terms of dynamic range and time optimization.

- Post-processing techniques can be applied to minimize the effect of reflections and noise.

- The technique is protected against phase drifts in the local oscillator, since the same LO is used for both channels (probe and reference). There are other phaseless solutions in the literature where this drift issue has already been pointed out [16].

- The tests performed show that the technique is valid for all the frequency range of the SDR. Therefore, radar signals, EMC or $5 \mathrm{G}$ devices are potential applications for this technique.

\section{SPHERICAL NEAR FIELD ANALYSIS}

If the position of the reference is optimized, the reference antenna is chosen properly and the receiver chain is adapted for the particular application, the uncertainties of the measurement can be minimized. A study is undergoing to optimize the measurement and determine the uncertainties introduced in amplitude and phase.

As a preliminary result, some error tests were carried out in the spherical near field multiprobe system at the StarLab in Satimo, Italy. The near-field acquisition of a SGH2000 was taken at 
2.3 GHz. The reference antenna used was a electric sleeve dipole. The measurements are compared with the VNA conventional measurements of the StarLab.

Considering the previous experience, the antenna was placed in a better position. More specifically, the reference is placed below the AUT, in that way, the antenna receives less power but it can be compensated with the receiver gain of the SDR. Moreover, the distance between the reference and the probes is increased which provides a more stable reference channel.

The scheme of the set-up can be seen in Fig. 10. In the multliprobe system the AUT rotates in azimuth, therefore the interference may be critical for probes in the shadow region of the arch, nevertheless these probes does not represent a significant part of the radiation of the device.

The near-field error between the electric-field measured by the proposed set-up and the conventional system has been compared by using Eq. (3). The electric-fields has been normalized to the maximum before the computation of the error.

$$
\operatorname{Error}(\mathrm{dB})=20 \log _{10}\left(E_{\text {conventional }}-E_{S D R}\right)
$$

The step chosen in $\theta$ and $\phi$ was $22.5^{\circ}$. That is traduced in 8 different azimuth positions and 15 different positions for $\theta$. Fig. 11 shows the errors for the different measurement points. It is clearly observed that the errors are mainly introduced for the probes in the shadow region and also when the AUT is rotated in azimuth the probes that are closer to the reference (from 1 to 6). Nevertheless, the mean error over the entire measurement surface is around $-38 \mathrm{~dB}$ which represents a very good starting point since post-processing techniques may even improve the final result as has been shown in Section 3.

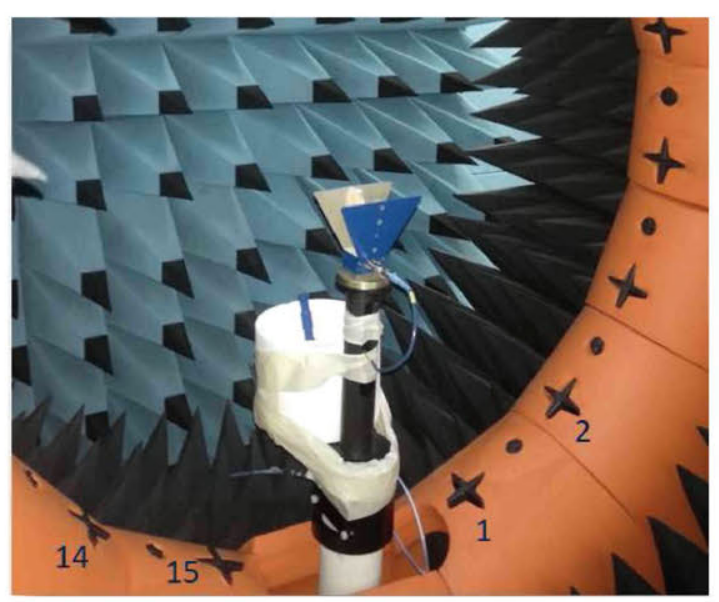

Figure 10: Spherical Near Field set-up.

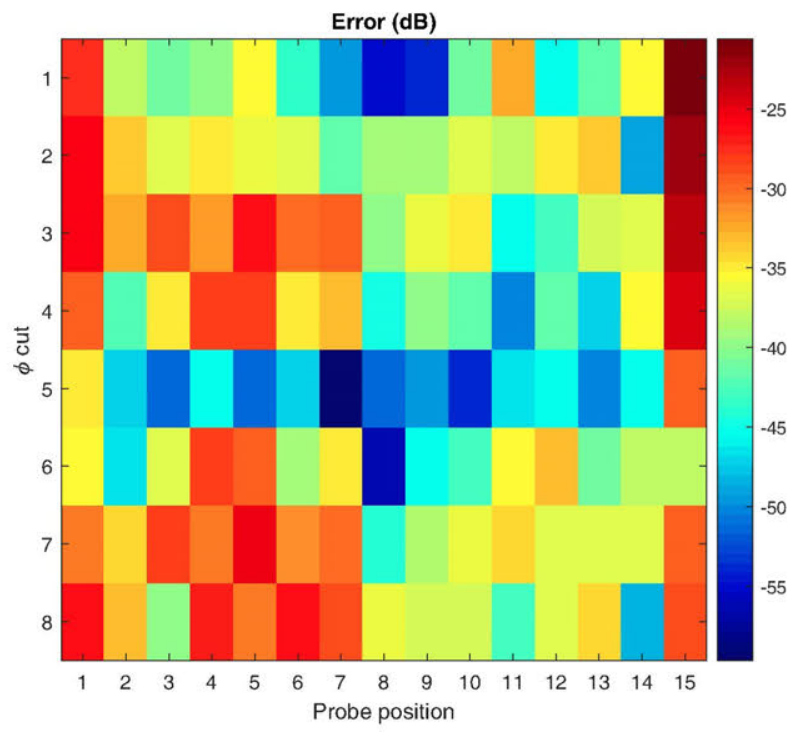

Figure 11: Spherical Near Field error (dB).

\section{CONCLUSIONS AND FUTURE LINES}

In this paper a new low-cost over-the-air antenna measurement technique by using a SDR receiver has been implemented and tested up to $6 \mathrm{GHz}$. The test has been performed in the planar near-field facility and some premiminary results has been presented for spherical near field measurements. The underlying idea of the method is based on evaluating the power of the probe antenna and calculating the phase of the AUT at each measurement point as the relative phase between one probe and a reference antenna. The results show a very good agreement between the expected radiation pattern and the measured one. The dynamic range of the SDR and the undesired reflections that may perturb the measurement due to the position of the reference antenna are the main limitations. Nevertheless, post-processing techniques and optimization of the reference remarkably improve the results obtained. The low-cost hardware necessary to implement the proposed technique is one of the main advantages in comparison with a conventional VNA. Moreover, the possibility of measuring antennas where the the reference signal is not accessible provides a set of advantages during the measurement process. 
As for future work, the evaluation of this technique should be tested for cases like EMC, antennas with an internal source or massive MIMO. This will prove a validation of this low-cost technique for scenarios where the phase is not accessible and will allow source reconstruction for antenna diagnostics. Furthermore, the quantitative evaluation of the uncertainties and optimization of the reference will provide a better understanding of the limitations of the system.

\section{ACKNOWLEDGMENT}

This work was supported by FUTURE-RADIO "Radio systems and technologies for high capacity terrestrial and satellite communications in an hyperconnected world" (project number TEC201785529-C3-1-R). The authors would like to acknowledge Microwave Vision Group (SATIMO) for the support during the measurements in the StarLab.

\section{REFERENCES}

1. Karim, M. R., X. Yang, and M. F. Shafique, "On chip antenna measurement: A survey of challenges and recent trends," IEEE Access, Vol. 6, 20320-20333, 2018.

2. Qi, Y., S. Member, G. Yang, L. Liu, and S. Member, "5G over-the-air measurement challenges: Overview," IEEE Transactions on Electromagnetic Compatibility, Vol. 59, No. 6, 1661-1670, 2017.

3. Leach, M. P., D. Smith, and S. P. Skobelev, "A modified holographic technique for planar near-field antenna measurements," IEEE Transactions on Antennas and Propagation, Vol. 56, No. 10, 2528-2532, 2008.

4. Yurduseven, O., D. Smith, B. Livingstone, V. Schejbal, and Z. You, "Investigations of resolution limits for indirect microwave holographic imaging," International Journal of RF and Microwave Computer-Aided Engineering, Vol. 23, No. 4, 410-416, 2013.

5. Laviada, J., Y. Avarez-Lopez, A. Arboleya-Arboleya, C. Garcia-Gonzalez, and F. Las-Heras, "Inverse scattering with phase retrieval based on indirect holography via synthesised planewaves," IET Microwaves, Antennas \& Propagation, Vol. 6, No. 12, 1389, 2012.

6. Schejball, V., V. Kovarik, and D. Cermak, "Synthesized-reference-wave holography for determining antenna radiation characteristics," IEEE Antennas and Propagation Magazine, Vol. 50, No. 5, 71-83, 2008.

7. Leach, M. P., D. Smith, S. P. Skobelev, and M. Elsdon, "An improved holographic technique for medium-gain antenna near field measurements," The Second European Conference on Antennas and Propagation, EuCAP 2007, Vol. 1, 1-6, Edinburgh, 2007.

8. Junkin, G., "Holographic testing of terahertz antennas," IEEE Transactions on Antennas and Propagation, Vol. 48, No. 3, 409-417, 2000.

9. Capozzoli, A., C. Curcio, G. D'Elia, and A. Liseno, "Millimeter-wave phaseless antenna characterization," IEEE Transactions on Instrumentation and Measurement, Vol. 57, No. 7, 13301337, 2008.

10. Puskely, J., "Application of iterative fourier method in cylindrical phaseless antenna measurement technique," Radioengineering, Vol. 21, No. 1, 422-429, 2012.

11. Schmidt, C. H. and Y. Rahmat-Samii, "Phaseless spherical near-field antenna measurements: Concept, algorithm and simulation," IEEE Antennas and Propagation Society, AP-S International Symposium (Digest), Vol. 2, 1-4, 2009.

12. Paulus, A., J. Knapp, and T. F. Eibert, "Utilizing partial knowledge of phase differences in convex optimization for amplitude-only near-field far-field transformation," 2017 11th European Conference on Antennas and Propagation, EUCAP 2017, 3766-3770, 2017.

13. Cano-Facila, F., S. Burgos, F. Martin, and M. Sierra-Castañer, "New reflection suppression method in antenna measurement systems based on diagnostic techniques," IEEE Transactions on Antennas and Propagation, Vol. 59, No. 3, 941-949, 2011.

14. Cano, F., M. Sierra-Castañer, and S. Burgos, "Novel method to improve the signal to noise ratio in the farfield results obtained from planar near-field measurements" IEEE Antennas and Propagation Magazine, Vol. 53, No. 2, 215-220, 2011.

15. Ludwig, A. C., "The definition of cross polarization," IEEE Transactions on Antennas and Propagation, Vol. 21, No. 1, 116-119, 1973.

16. Brockett, T. and Y. Rahmat-Samii, "Measuring the radiation pattern of integrated antennas with internal sources: A phaseless near-field technique," IEEE Antennas and Propagation Society, AP-S International Symposium (Digest), 2257-2258, 2013. 